\title{
Ambiguities in a Problem in Planar Geodesy
}

Josef SCHICHO and Matteo GALLET

Research Institute for Symbolic Computation, Johannes Kepler University, Altenberger Strasse 69, 4040 Linz, Austria

E-mail: josef.schicho@risc.jku.at,mgallet@risc.jku.at

Received October 29, 2014, in final form January 16, 2015; Published online January 27, 2015

http://dx.doi.org/10.3842/SIGMA.2015.008

\begin{abstract}
This is a study of a problem in geodesy with methods from complex algebraic geometry: for a fixed number of measure points and target points at unknown position in the Euclidean plane, we study the problem of determining their relative position when the viewing angles between target points seen from measure points are known. In particular, we determine all situations in which there is more than one solution.
\end{abstract}

Key words: surveying; structure and motion problem; Gale duality

2010 Mathematics Subject Classification: 14N05; 14N15

\section{Introduction}

Let $t>0$ and $m>0$ be integers. We consider the problem of identifying the relative position of $t+m$ unknown points $p_{1}, \ldots, p_{t}, q_{1}, \ldots, q_{m}$ in the Euclidean plane from the angles $\measuredangle_{q_{j}}\left(p_{i}, p_{k}\right)$, for $1 \leq i<k \leq t$ and $1 \leq j \leq m$. We call $p_{1}, \ldots, p_{t}$ the target points and $q_{1}, \ldots, q_{m}$ the measure points (see Fig. 1).

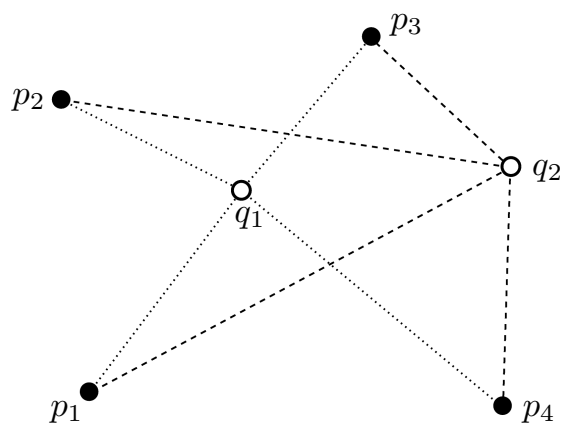

Figure 1. An example with $t=4$ and $m=2$, namely with four target points $p_{1}, \ldots, p_{4}$ and two measure points $q_{1}, q_{2}$.

Apparently, all angles can be computed from the angles $\measuredangle_{q_{j}}\left(p_{1}, p_{k}\right)$ for $2 \leq k \leq t$ and $1 \leq j \leq m$, since $\measuredangle_{q_{j}}\left(p_{i}, p_{k}\right)=-\measuredangle_{q_{j}}\left(p_{1}, p_{i}\right)+\measuredangle_{q_{j}}\left(p_{1}, p_{k}\right)$. So the dimension of given data is $m(t-1)$, and the dimension of the unknown data is $2(m+t-2)$; in order that the problem is not undetermined, we should have

$$
0 \leq m(t-1)-2(m+t-2)=(m-2)(t-3)-2 .
$$

In the two cases where equality holds, there are in general two solutions, namely there exist two $(m+t)$-tuples of points in the plane both fitting with the data about the angles.

To explain the situation for $t=m=4$, we introduce the profile of a fixed sequence $\vec{p}$ of target points, as the two-dimensional set of all possible measurement results from various measure points. Then we decompose our problem into two steps. First, we interpolate the profile 
surface from the given angles. Once the profile is determined, additional measure points (and the corresponding measured angles) do not reveal any more information on the position of the target points. Then we determine the target points from the profile. Remarkably, it turns out that for target sequences $\vec{p}$ with $t=4$, there is in general a second sequence $\vec{p}^{\prime}$ with the same profile.

Similarly, we treat the case $m=3$ and $t=5$ by introducing the co-profile of a sequence $\vec{q}$ of measure points, namely the two-dimensional set of measurements of target points from $\vec{q}$; however, the situation is not entirely symmetric to the case of the profile because here we need in addition one target point in order to define the co-profile. In this case, additional target points (and the corresponding measured angles) do not reveal any more information on the position of the measure points and the prescribed target point.

Section 2 is devoted to the introduction of profile and co-profile for arbitrary $t$ and $m$. In Section 3, we determine the situations where the profile or the co-profile cannot be uniquely determined, also paying attention to non-general situations. In Section 4 we identify the target points once the profile is known. We determine the situations where this is not uniquely possible. Once we know the target points, the determination of the measure points from the profile is not problematic. The complete discussion is summarized in Theorem 4.6.

Strictly speaking, the two solutions for the general case $m=t=4$ cannot be distinguished algebraically, by means of testing polynomial equalities. But angle measurement also gives information corresponding to inequalities, namely we can detect on which of the two rays of a line a certain target point lies. Taking this additional information into account, one can distinguish the two solutions in many cases, but not in all of them. This aspect is discussed in more detail in Section 5.

The majority of the results in this paper is not new. The stated problem is equivalent to the reconstruction of a set of points in the plane from images by a one-dimensional calibrated camera. The paper [1] (also contained, in expanded form, in [9]) explains the two solutions for the case $m=3$ by a theorem of planar geometry. There the authors also describe a duality exchanging the role of measure points and target points, except for one target point that has to keep its role as a target point; using this duality, the existence of two solutions for $t=4$ is explained as well. A similar duality is also known for the uncalibrated case (see [10]), where three fixed target points keep their role instead of one, and also for two-dimensional pictures of points in 3-space (see [2]). The two solutions for the general case appear also in the one-dimensional uncalibrated case in [10]; indeed, the calibrated case can be reduced to the uncalibrated case by considering the two cyclic points of the Euclidean plane as additional target points. The classification of exceptional cases with infinitely many solutions in Theorem 4.6 seems to be new, as well as the discussion of ambiguities taking direction information into account (Theorem 5.4). However, we think that our uniform discussion of the ambiguities has some value. Also, the results are obtained by general theorems in algebraic geometry, without technical computations; a single exception is Remark 4.5, which is not used in the remaining part of the paper.

\section{Algebraic description of given data}

Notation. Throughout the paper we will often use the adjective general talking about points or measurements: as it is often done in algebraic geometry, here we mean that a property is general if it holds for a Zariski open set of a space parametrizing the objects we are interested in. Hence when we say that a property holds for "general $t$ target points $\vec{p}$ and $m$ measure points $\vec{q}$ " we mean that the set of pairs $(\vec{p}, \vec{q})$ for which the property does not hold is a variety of dimension less than $2(m+t)$.

The aim of this section is to associate to any given tuple $\vec{p}$ of target points a projective variety, called the profile of $\vec{p}$, and to any given tuple $\vec{q}$ of measure points together with a prescribed 
target point $p_{1}$ another projective variety, called the co-profile of $\vec{q}$ and $p_{1}$. These two varieties encode information about, respectively, all possible measurements of $\vec{p}$ from any measure points, and measurements with respect to $\vec{q}$ and $p_{1}$ of any possible target point.

Notation. For target points, we use real coordinates $(u, v)$, and we introduce complex coordinates $w=u+\mathrm{i} v$ and $\bar{w}=u-\mathrm{i} v$. For measure points, we use real coordinates $(x, y)$, and we introduce complex coordinates $z=x+\mathrm{i} y$ and $\bar{z}=x-\mathrm{i} y$.

The first aspect to clarify is how we model measurements, namely angles. We decide to model them by complex numbers with modulus 1 . More precisely, if the measure point $q$ has complex coordinate $z$ and the target points $p_{1}$ and $p_{2}$ have complex coordinates $w_{1}$ and $w_{2}$, one can check that the angle $\measuredangle_{q}\left(p_{1}, p_{2}\right)$ is given by the complex number $\frac{\left(\bar{z}-\bar{w}_{1}\right)\left(z-w_{2}\right)}{\left(\bar{z}-\bar{w}_{1}\right)\left(z-w_{2}\right) \mid}$. We fix now pairwise distinct target points $p_{1}, \ldots, p_{t}$ (with real/complex coordinates as above). Hence we can define a map $\mathbb{R}^{2} \backslash\left\{p_{1}, \ldots, p_{t}\right\} \longrightarrow \mathbb{C}^{t-1}$ sending

$$
z=x+\mathrm{i} y \mapsto\left(\frac{\left(\bar{z}-\bar{w}_{1}\right)\left(z-w_{2}\right)}{\left|\left(\bar{z}-\bar{w}_{1}\right)\left(z-w_{2}\right)\right|}, \ldots, \frac{\left(\bar{z}-\bar{w}_{1}\right)\left(z-w_{t}\right)}{\left|\left(\bar{z}-\bar{w}_{1}\right)\left(z-w_{t}\right)\right|}\right) .
$$

This map is far from being algebraic. In order to make it algebraic, we start by squaring each coordinate of the image (this corresponds to multiplying all angles by a factor of 2) and then homogenize with respect to a new variable, which we place in first position, so that the domain becomes a projective space. What we obtain has the following expression, which still involves complex conjugation, so it is not yet what we are looking for:

$$
\begin{aligned}
& f_{\vec{p}}: \mathbb{R}^{2} \backslash\left\{p_{1}, \ldots, p_{t}\right\} \longrightarrow \mathbb{P}_{\mathbb{C}}^{t-1}, \\
& z \mapsto\left(F_{1}(z): \ldots: F_{t}(z)\right), \\
& F_{1}(z)=\left(z-w_{1}\right)\left(\bar{z}-\bar{w}_{2}\right) \cdots\left(\bar{z}-\bar{w}_{t}\right) \text {, } \\
& F_{2}(z)=\left(\bar{z}-\bar{w}_{1}\right)\left(z-w_{2}\right) \cdots\left(\bar{z}-\bar{w}_{t}\right) \text {, } \\
& F_{t}(z)=\left(\bar{z}-\bar{w}_{1}\right)\left(\bar{z}-\bar{w}_{2}\right) \cdots\left(z-w_{t}\right) .
\end{aligned}
$$

We proceed towards our goal to obtain an algebraic map, and in particular we want to make $f_{\vec{p}}$ into a rational map between projective varieties. To do so we choose the following injection ${ }^{1}$ of $\mathbb{R}^{2}$ into $\mathbb{P}_{\mathbb{C}}^{1} \times \mathbb{P}_{\mathbb{C}}^{1}$ :

$$
\begin{aligned}
& \mathbb{R}^{2} \longrightarrow \mathbb{P}_{\mathbb{C}}^{1} \times \mathbb{P}_{\mathbb{C}}^{1}, \\
& (x, y) \mapsto \underbrace{(x+\mathrm{i} y: 1) \times(x-\mathrm{i} y: 1)}_{=(z: 1) \times(\bar{z}: 1)} .
\end{aligned}
$$

Then we can extend the previously defined map $f_{\vec{p}}$ to a map from $\mathbb{P}_{\mathbb{C}}^{1} \times \mathbb{P}_{\mathbb{C}}^{1}$, which we still denote by $f_{\vec{p}}$. If we take coordinates $\left(\alpha_{1}: \beta_{1}\right) \times\left(\alpha_{2}: \beta_{2}\right)$ on $\mathbb{P}_{\mathbb{C}}^{1} \times \mathbb{P}_{\mathbb{C}}^{1}$, the new map is given by

$$
\begin{aligned}
f_{\vec{p}}: & \mathbb{P}_{\mathbb{C}}^{1} \times \mathbb{P}_{\mathbb{C}}^{1} \rightarrow \mathbb{P}_{\mathbb{C}}^{t-1}, \\
& \left(\alpha_{1}: \beta_{1}\right) \times\left(\alpha_{2}: \beta_{2}\right) \mapsto\left(G_{1}(\alpha, \beta): \ldots: G_{t}(\alpha, \beta)\right) .
\end{aligned}
$$

The components $G_{j}$ of $f_{\vec{p}}$ are obtained from the previously defined polynomials $F_{j}$ by substituting all factors $\left(z-w_{i}\right)$ with $\left(\alpha_{1}-\beta_{1} w_{i}\right)$ and all factors $\left(\bar{z}-\bar{w}_{i}\right)$ with $\left(\alpha_{2}-\beta_{2} \bar{w}_{i}\right)$. Thus we see that this is a rational map between complex projective varieties.

\footnotetext{
${ }^{1}$ The choice of $\mathbb{P}_{\mathbb{C}}^{1} \times \mathbb{P}_{\mathbb{C}}^{1}$, instead of, for example, $\mathbb{P}_{\mathbb{C}}^{2}$, will be justified a posteriori by the fact that in this way we will be able to prove some properties of the map $f_{\vec{p}}$ in an easier way.
} 
Remark 2.1. Note that, since during the construction of $f_{\vec{p}}$ we take the squares of the involved quantities (this corresponding to multiplication by 2 of the angles), we have that $f_{\vec{p}}(q)$ determines the measured angles only modulo $\pi$. This is a consequence of the use of complex numbers: since complex numbers are not ordered, the "complex-valued" part of our angle measurement device is unable to tell two opposite directions of the same line apart.

Remark 2.2. If we remove the last target point and we consider $\overrightarrow{p^{*}}=\left(p_{1}, \ldots, p_{t-1}\right)$, then the map $f_{\overrightarrow{p^{*}}}$ is equal to $f_{\vec{p}}$ composed with the projection $\mathbb{P}_{\mathbb{C}}^{t-1} \rightarrow \mathbb{P}_{\mathbb{C}}^{t-2}$ given by $\left(s_{1}: \ldots: s_{t}\right) \mapsto\left(s_{1}\right.$ : $\left.\ldots: s_{t-1}\right)$.

Definition 2.3. The Zariski closure of the image of the map $f_{\vec{p}}$ is an algebraic surface $S_{\vec{p}} \subset \mathbb{P}_{\mathbb{C}}^{t-1}$, which we call the profile of $\vec{p}$.

Proposition 2.4. If $t \geq 3$, then the measurement map $f_{\vec{p}}$ is a birational map from the plane to the profile of $\vec{p}$, for any point sequence $\vec{p}$ of pairwise distinct points.

Proof. First, assume that $t=3$. We recall a well-known (for surveying or land navigation purposes; see for example [8]) geometric construction of a left inverse of the measurement map: for each $q \in \mathbb{R}^{2}$, the oriented angle $\measuredangle_{q}\left(p_{1}, p_{2}\right)$ modulo $\pi$ determines a circle on which $q$ lies. Similarly, the oriented angle $\measuredangle_{q}\left(p_{1}, p_{3}\right)$ modulo $\pi$ determines another circle. The two circles intersect in $p_{1}$ and in a second point, which must be $q$. It is straightforward to express this geometric construction as a rational map in the angles.

For $t>3$, a left inverse can be given as the projection to the first three coordinates followed by the inverse for the case $t=3$ above.

Notation. The inverse of the measurement map is called the resection map.

Remark 2.5. From the geometric construction above, it is clear that the measurement map is injective outside the circle through any triple of points $p_{i}, p_{j}, p_{k}$ (or the line through $p_{i}, p_{j}, p_{k}$ if the points are collinear).

Here is a description of the profile.

Lemma 2.6. If $t \geq 3$, then the profile surface $S_{\vec{p}} \subset \mathbb{P}_{\mathbb{C}}^{t-1}$ has degree $t-2$ and passes through the $t+1$ points $(1: \ldots: 0), \ldots,(0: \ldots: 1),(1: \ldots: 1)$. Its ideal is generated by the $2 \times 2$ minors of a $2 \times(t-2)$ matrix whose entries are linear forms.

Proof. The functions $G_{1}, \ldots, G_{t}$ have bidegree $(1, t-1)$. The base locus of $f_{\vec{p}}$ consists of $t$ simple base points corresponding to $p_{1}, \ldots, p_{t}$, hence the degree of the image is $2(t-1)-t=t-2$. For an explanation regarding the previous formula we refer to $[3, \text { Appendix } \mathrm{A}]^{2}$. For $r \in\{1, \ldots, t\}$, the image of the line $\alpha_{2}-\beta_{2} \bar{w}_{r}$ is the point $(0: \ldots: 1: \ldots: 0)$ (with 1 at position $r$ ); the point $(1: \ldots: 1)$ appears as the image of the point at infinity $(1: 0) \times(1: 0)$.

Because $\operatorname{deg}\left(S_{\vec{p}}\right)=\operatorname{codim}\left(S_{\vec{p}}\right)+1$, we have a surface of minimal degree. Such a surface is determinantal, and the generators of its ideal are well-known (see for example [5]).

We come to the definition of the co-profile: for this purpose let us fix a tuple $\vec{q}=\left(q_{1}, \ldots, q_{m}\right)$ of measure points and a target point $p_{1}$. We consider the map $\mathbb{R}^{2} \longrightarrow \mathbb{C}^{m}$ associating to each

\footnotetext{
${ }^{2}$ Here the author clarifies [3, equation (5.1)] using arguments from intersection theory. The only difference between our formula and [3, equation (5.1)] is that in the latter one considers homogeneous polynomials of degree $n$, and this justifies the term $n^{2}$ appearing there, while in our formula we have bihomogeneous polynomials of bidegree $(1, t-1)$, yielding the term $2(t-1)$. The term $t$ in our formula, given by the $t$ simple base points, corresponds to the term $\sum_{p \in Z} e\left(I_{Z, p}, \mathcal{O}_{\mathbb{P}^{2}, p}\right)$ in [3, equation (5.1)].
} 
target point $p \in \mathbb{R}^{2}$ the $m$-tuple of angles $\left(\measuredangle_{q_{1}}\left(p_{1}, p\right), \ldots, \measuredangle_{q_{m}}\left(p_{1}, p\right)\right)$. Performing analogous homogeneization and operations as before we obtain a rational map:

$$
\begin{aligned}
f_{\vec{q}, p_{1}}^{\prime}: & \mathbb{P}_{\mathbb{C}}^{1} \times \mathbb{P}_{\mathbb{C}}^{1} \rightarrow \mathbb{P}_{\mathbb{C}}^{m}, \\
& \left(\alpha_{1}: \beta_{1}\right) \times\left(\alpha_{2}: \beta_{2}\right) \mapsto\left(G_{0}^{\prime}(\alpha, \beta): \ldots: G_{m}^{\prime}(\alpha, \beta)\right)
\end{aligned}
$$

Definition 2.7. The Zariski closure of the image of the map $f_{\vec{q}, p_{1}}^{\prime}$ is an algebraic surface $S_{\vec{q}, p_{1}}^{\prime} \subset$ $\mathbb{P}_{\mathbb{C}}^{m}$, which we call the co-profile of $\vec{q}$ and $p_{1}$.

Lemma 2.8. If $m \geq 2$, then the co-profile map $f_{\vec{q}, p_{1}}^{\prime}$ is birational, and the co-profile surface $S_{\vec{q}, p_{1}}^{\prime} \subset \mathbb{P}_{\mathbb{C}}^{m}$ has degree $m-1$ and passes through the $m+1$ points $(1: \ldots: 0), \ldots,(0: \ldots: 1),(1$ : $\ldots: 1)$. Its ideal is generated by the $2 \times 2$ minors of $a 2 \times(m-1)$ matrix whose entries are linear forms.

Proof. For the inverse of $f_{\vec{q}, p_{1}}^{\prime}$, there is again a geometric construction: when we know $\measuredangle_{q_{1}}\left(p_{1}, p\right)$ and $\measuredangle_{q_{2}}\left(p_{1}, p\right)$, then we can construct $p$ by intersecting the two lines determined by the angles above.

The proof of the statement on $S_{\vec{q}, p_{1}}^{\prime}$ is analogous to the proof of Lemma 2.6, with some changes in the details: the functions $G_{0}^{\prime}, \ldots, G_{m}^{\prime}$ have bidegree $(1, m)$, the base points correspond to $q_{1}, \ldots, q_{m}$ and the point at infinity $(1: 0) \times(1: 0)$; the images of the exceptional lines are the points $(1: \ldots: 0), \ldots,(0: \ldots: 1)$, and the point $(1: \ldots: 1)$ appears as the image of $p_{1}$.

\section{An interpolation problem}

As remarked in the Introduction, we are going to solve our problem in two steps. The first step, discussed in this section, is to compute the profile of a tuple $\vec{p}$ from a fixed number $m$ of measurements. The task is to find a surface of known degree, and we know $m+t+1$ points on the surface, namely $m$ points from measurements and $t+1$ points from Lemma 2.6. We call this step profile interpolation. Once the profile is computed, we know all possible measurements up to $\pi$, hence additional values obtained with the "complex angle measurement device", namely the map $f_{\vec{p}}$, do not provide more information. Analogously, one can try to compute the co-profile of $\vec{q}$ and $p_{1}$ from other $t-1$ target points. We will see that the solution of this problem is not always unique, namely there may exist more than one profile/co-profile matching the given data.

If we are given $t$ target points $\vec{p}$ and $m$ measure points $\vec{q}$, then we can form an $m \times(t-1)$ matrix $M_{\vec{p}, \vec{q}}$, called the double angle matrix:

$$
M_{\vec{p}, \vec{q}}=\left(\begin{array}{ccc}
\left(\measuredangle_{q_{1}}\left(p_{1}, p_{2}\right)\right)^{2} & \ldots & \left(\measuredangle_{q_{1}}\left(p_{1}, p_{t}\right)\right)^{2} \\
\vdots & & \vdots \\
\left(\measuredangle_{q_{m}}\left(p_{1}, p_{2}\right)\right)^{2} & \ldots & \left(\measuredangle_{q_{m}}\left(p_{1}, p_{t}\right)\right)^{2}
\end{array}\right) .
$$

In this way the rows of $M_{\vec{p}, \vec{q}}$ are the input for profile identification, while the columns of $M_{\vec{p}, \vec{q}}$ are the input for co-profile identification.

We start with the case $t=4$. Here the profile is a quadric surface in $\mathbb{P}_{\mathbb{C}}^{3}$.

Proposition 3.1. If $t=4$, then the profile can be computed from 4 measurements, in general.

Proof. The linear space of quadratic forms in 4 variables is 10-dimensional, and each point gives a linear condition on it. For general measurements, the 4 points in $S_{\vec{p}}$ from the measurements and the 5 points from Lemma 2.6 give linear independent conditions, and so there is a onedimensional solution space which determines the profile uniquely. 
Remark 3.2. If $t>4$, then it is also possible to compute the profile from 4 general measurements. For instance, if $\vec{p}=\left(p_{1}, \ldots, p_{5}\right)$, then we can compute the profiles $S_{1}$ and $S_{2}$ of $\left(p_{1}, p_{2}, p_{3}, p_{4}\right)$ and of $\left(p_{1}, p_{2}, p_{3}, p_{5}\right)$, respectively. Then the profile of $\vec{p}$ is the closure of the set

$$
\left\{\left(x_{0}: x_{1}: x_{2}: x_{3}: x_{4}\right) \in \mathbb{P}_{\mathbb{C}}^{4} \mid\left(x_{0}: x_{1}: x_{2}: x_{3}\right) \in S_{1} \text { and }\left(x_{0}: x_{1}: x_{2}: x_{4}\right) \in S_{2}\right\} .
$$

As the parameter counting argument in the Introduction suggests, we can remove one measure point if $t \geq 5$ and still have a finite number of solutions. Here is the precise statement.

Proposition 3.3. For $t=5$ and $m=3$, there are in general two solutions for the profile interpolation problem.

Proof. In this case the profile is a rational cubic surface in $\mathbb{P}_{\mathbb{C}}^{4}$. By [6, Example 3.4], there are exactly two such cubic surfaces passing through 9 points in general position in $\mathbb{P}_{\mathbb{C}}^{4}$. Interestingly, the proof there uses Gale duality, which seems to be tightly related to the correspondence between the $m$ points on the profile and the $t-1$ points on the co-profile given by a double angle matrix $M_{\vec{p}, \vec{q}}$ : in both cases, in fact, the coordinates of the two sets of points are given by the rows of a matrix and its transpose.

Intuitively, increasing $t$ should increase the amount of information, so we may think that for $t>5$ and $m=3$, we have only a single solution for the profile interpolation problem. We will see later that this is not the case. What is true is that considering also the co-profile increases the amount of insight.

Proposition 3.4. If $m=3$ and $t \geq 5$, then the co-profile can be uniquely computed, in general.

Proof. The statement is analogous to Proposition 3.1: again, we have to interpolate 9 or more points via a quadric surface in $\mathbb{P}_{\mathbb{C}}^{3}$.

Proposition 3.5. For $t=4$ and $m=4$, there are in general two solutions for the co-profile interpolation problem.

Proof. Here the situation is as in Proposition 3.3: we have to interpolate 9 points using cubic surfaces in $\mathbb{P}_{\mathbb{C}}^{4}$.

Proposition 3.6. For $m=3$ and $t>5$, there are in general two solutions for the profile interpolation problem. Dually, for $m>4$ and $t=4$, there are in general two solutions for the co-profile interpolation problem.

Proof. If $m=3$ and $t=5$, then we know two possible candidates for the profile, giving two possible candidates for the position of measure/target points; however the two co-profiles must be equal by Proposition 3.4. But if we know the co-profile, then any measurements from new target points does not give any additional information about the position of the old target points. Therefore we will never be able to tell the true profile, no matter how many new target points we introduce.

The proof of the dual statement is analogous.

So far we completed the description of the profile/co-profile interpolation in the general case, and we summarize the results in Theorem 3.7.

Theorem 3.7. Let $\vec{p}$ and $\vec{q}$ be general $t$ and $m$-tuples of points in $\mathbb{R}^{2}$. Then the following tables summarize the number of possible profiles and co-profiles compatible with the double angle matrix $M_{\vec{p}, \vec{q}}$ : 
Table 1. Number of profiles fitting, in general, a given double angle matrix.

\begin{tabular}{c|ccc}
$m \backslash t$ & 3 & 4 & 5 or more \\
\hline 3 & 1 & $\infty$ & 2 \\
4 or more & 1 & 1 & 1
\end{tabular}

Table 2. Number of co-profiles fitting, in general, a given double angle matrix.

\begin{tabular}{c|ccc}
$m \backslash t$ & 3 & 4 & 5 or more \\
\hline 3 & $\infty$ & $\infty$ & 1 \\
4 or more & $\infty$ & 2 & 1
\end{tabular}

In Theorem 3.10 we analyze those cases for which for a general choice of the points $\vec{p}$ and $\vec{q}$ there is a unique profile or co-profile, but for some special choices there may be more than one profile/co-profile, and we identify how these special choices look like. Considering Tables 1 and 2 we see that, in general, we have uniqueness for the profile if $m=t=3$ or if $m \geq 4$ and $t \geq 4$, and for the co-profile if $m \geq 3$ and $t \geq 5$. Notice that if $t=3$, then the profile is equal to $\mathbb{P}_{\mathbb{C}}^{2}$, and so in this case it can never happen, even in special situations, that the profile is not unique.

Notation. A cyclic cubic curve is a plane cubic curve passing through the two cyclic points at infinity, with homogeneous coordinates $(0: 1: \pm \mathrm{i})$. In the embedding of $\mathbb{R}^{2}$ into $\mathbb{P}_{\mathbb{C}}^{1} \times \mathbb{P}_{\mathbb{C}}^{1}$ that we use, the equation of a cyclic curve has bidegree $(2,2)$ and passes through the point $(1: 0) \times(1: 0)$.

We allow cyclic cubics to be reducible. There are two reducible cases: a line and a circle, or a conic and the line at infinity.

We start treating the base cases.

Lemma 3.8. Suppose that $t=4$ and $m \geq 4$ and that the profile is not uniquely determined by the double angle matrix $M_{\vec{p}, \vec{q}}$, or that $t \geq 5$ and $m=3$ and the co-profile is not uniquely determined by the double angle matrix $M_{\vec{p}, \vec{q}}$. Then there exists a cyclic cubic curve that contains all target points and all measure points, or a line that contains all target points.

Proof. First, assume $t=4$ and $m \geq 4$, so that the profile surface $S$ is a quadric surface in $\mathbb{P}_{\mathbb{C}}^{3}$. Moreover we assume that the profile is not unique. This is equivalent to the fact that the $m+5$ points $f_{\vec{p}}\left(q_{1}\right), \ldots, f_{\vec{p}}\left(q_{m}\right)$ and $(1: \ldots: 0), \ldots,(0: \ldots: 1)$ and $(1: \ldots: 1)$ are contained in the intersection with a second quadric $Q$. The pullback under $f_{\vec{p}}$ of any plane section of $S$ is a divisor of $\mathbb{P}_{\mathbb{C}}^{1} \times \mathbb{P}_{\mathbb{C}}^{1}$ of bidegree $(1,3)$ passing through $p_{1}, \ldots, p_{4}$, therefore the pullback of any quadric section is a divisor of bidegree $(2,6)$ passing with multiplicity at least 2 through $p_{1}, \ldots, p_{4}$. In addition, by hypothesis $Q$ passes through the point $(1: \ldots: 0)$, which is the image of an exceptional divisor $E_{1}$, namely the unique curve of bidegree $(0,1)$ passing through $p_{1}$. Hence the pullback of $Q$ contains this divisor $E_{1}$. Similarly, the pullback contains the other 3 exceptional divisors $E_{2}, E_{3}$ and $E_{4}$. If we remove these components, it remains a curve of bidegree $(2,2)$ passing through $p_{1}, \ldots, p_{4}$. It must contain the preimage of $(1: \ldots: 1)$, which is the point $(1: 0) \times(1: 0)$, and all measure points $q_{1}, \ldots, q_{m}$. So we have found a cyclic cubic curve as stated in the thesis.

The proof for the dual assertion assuming non-uniqueness of the co-profile is similar.

Example 3.9. Consider the case $t=m=4$ where all 4 target points $p_{1}, \ldots, p_{4}$ lie on a circle and the 2 measure points $q_{1}$ and $q_{2}$ lie on the same circle, as in Fig. 2. In this case all 8 points lie on a (reducible) cyclic cubic, given by the union of the circle and a line.

Since all $p_{1}, \ldots, p_{4}, q_{1}$ and $q_{2}$ lie on the same circle, we have the equalities

$$
\measuredangle_{q_{1}}\left(p_{1}, p_{k}\right)=\measuredangle_{q_{2}}\left(p_{1}, p_{k}\right) \quad \text { for all } k \in\{2,3,4\},
$$

which imply that the conditions imposed by $q_{1}$ and $q_{2}$ to the linear space of quadratic forms in 4 variables as in the proof of Proposition 3.1 are not independent. This means that there exists at least a two-dimensional linear space of such forms, this ensuring the existence of infinitely many possible profiles for the pair $(\vec{p}, \vec{q})$. 


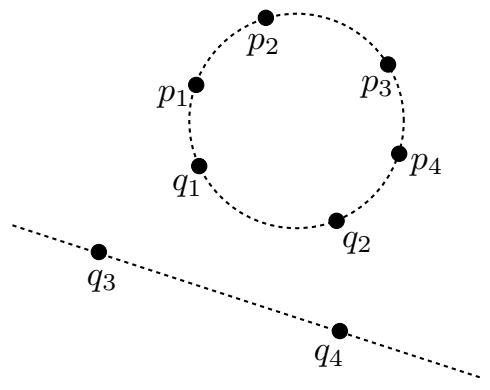

Figure 2. An example of a configuration of target and measure points for which the profile is not unique: the four target points lie on a circle, two measure points lie on the same circle, and the other two measure points lie on a line.

It would be nice now to use an inductive argument (on the number of target or measure points) to establish the same result as in Lemma 3.8 for all remaining cases. Unfortunately, in each induction step we encounter new possibilities, making a uniform description difficult to achieve. Because of this, we prefer to strengthen the hypotheses of our theorem in order to get rid of these "spurious" cases.

Theorem 3.10. Assume that, for given sequences $\vec{p}$ of target points and $\vec{q}$ of measure points, either $m \geq 4$ and the profile is not uniquely determined by the double angle matrix $M_{\vec{p}, \vec{q}}$, or $t \geq 5$ and the co-profile is not uniquely determined by the double angle matrix $M_{\vec{p}, \vec{q}}$. Assume furthermore that no 5 target points and no 4 measure points are cocircular or collinear. Then there exists a cyclic cubic curve that contains all target points and all measure points.

Proof. Let us start analyzing the case when $t=5$ and $m \geq 4$. We try to reduce to Lemma 3.8. By hypothesis we know that the profile is not unique. If now we consider any subset $\vec{p}^{\prime}$ of four points out of the five points of $\vec{p}$, we have that the profile of $\vec{p}^{\prime}$ and $\vec{q}$ may or may not be unique. Notice that if we could find two four tuples $\vec{p}^{\prime}$ and $\vec{p}^{\prime \prime}$ for which the profile is unique, then by Remark 3.2 we would be able to reconstruct the profile of $\vec{p}$ and $\vec{q}$ uniquely, and this is against the assumption. Hence there can be at most one 4-subtuple $\vec{p}^{\prime}$ for which the profile is unique. So we can distinguish two cases:

Case $i$. For all 4 -subtuples $\vec{p}^{\prime}$, the profile is not uniquely determined. Then by Lemma 3.8 we have that all points $\vec{p}^{\prime}$ and $\vec{q}$ lie on a cyclic cubic for each $\vec{p}^{\prime}$. We can consider the linear system of all cyclic cubics through $q_{1}, \ldots, q_{m}$. It defines a rational map $g:\left(\mathbb{P}_{\mathbb{C}}^{1} \times \mathbb{P}_{\mathbb{C}}^{1}\right)-\rightarrow \mathbb{P}_{\mathbb{C}}^{N}$, where $N$ is the rank of the linear system (namely its dimension as a projective space). Since 4 real points in the plane give independent conditions for interpolating a cyclic cubic, it follows that $N \leq 3$. Suppose now that the linear system giving $g$ does not have any fixed component. Then because any four target points lie on cyclic cubic, it follows that any four of their images under $g$ lie on hyperplane. Hence by a linear algebra argument all images of the target points lie on a hyperplane, and this hyperplane corresponds to a cyclic cubic curve as stated in the thesis. If the linear system has some fixed component then this fixed component can only be a circle or a line, and this contradicts the hypothesis, since we supposed that no 4 measure points are cocircular or aligned.

Case ii. There exists a 4-subtuple $\vec{p}^{\prime}$ for which the profile is unique, but for any other 4subtuple $\vec{p}^{\prime \prime}$ the profile is not unique. After possibly relabeling the points we can assume that $\vec{p}^{\prime}=\left(p_{2}, p_{3}, p_{4}, p_{5}\right)$. We can define a map $g:\left(\mathbb{P}_{\mathbb{C}}^{1} \times \mathbb{P}_{\mathbb{C}}^{1}\right) \rightarrow \mathbb{P}_{\mathbb{C}}^{N}$ as in Case i. Suppose that the linear system $\Lambda$ of cyclic cubics through $q_{1}, \ldots, q_{m}$ does not have any fixed component. Then by hypothesis we have that each 4-tuple of points $\left(g\left(p_{1}\right), g\left(p_{i}\right), g\left(p_{j}\right), g\left(p_{k}\right)\right)$ lies on the same plane for all $i, j, k \in\{2,3,4,5\}$. This forces $g\left(p_{2}\right), \ldots, g\left(p_{5}\right)$ to lie on the same plane, but this contradicts the hypothesis, since the profile of $\vec{p}^{\prime}=\left(p_{2}, p_{3}, p_{4}, p_{5}\right)$ is unique. Hence the 
linear system $\Lambda$ has a fixed component, but this means that the measure points are collinear or cocircular. Hence under our hypothesis Case ii never arises.

If now $t$ is arbitrary, we can argue as before showing that at most one $t-1$ tuple of target points admits a unique profile, and use induction on $t$ since the hypothesis ensures that Case ii never happens.

The proof for the dual assertion assuming non-uniqueness of the co-profile is similar, but with one modification. If $m \geq 4$ and $t \geq 5$, then the linear system of cyclic cubics through all target points defines the rational map $g:\left(\mathbb{P}_{\mathbb{C}}^{1} \times \mathbb{P}_{\mathbb{C}}^{1}\right) \rightarrow \mathbb{P}_{\mathbb{C}}^{N}$, where $N$ is the rank of the linear system (which, in this case, is less than or equal to 2). Here is the modification: 5 real points may fail to give independent conditions for interpolating a cyclic cubic, and this happens if and only if the all target points are collinear. However this situation is ruled out by the hypothesis, so we have the thesis.

\section{Point identification}

We are turning to the task of determining the target points $p_{1}, \ldots, p_{t}$, assuming we already know the surface $S_{\vec{p}} \subset \mathbb{P}_{\mathbb{C}}^{t-1}$, describing all possible results of measurements modulo $\pi$. This problem can only be solved up to similarities, namely rotations, translations and dilations. For $t=3$, the problem is clearly not solvable, because in that case $S_{\vec{p}}=\mathbb{P}_{\mathbb{C}}^{2}$ does not give any information on the target points.

Proposition 4.1. If $t \geq 5$ and the target points are not collinear, then the point identification problem has a unique solution.

Proof. One sees that it is enough to prove the statement for $t=5$. To do this, one can proceed algebraically, showing that point identification boils down to solving systems of linear equations admitting in general a unique solution. Otherwise one can use the following argument. We assume that no 4 points are collinear. If we consider four points $p_{i}, p_{j}, p_{k}, p_{h}$ out of the five, there exists a unique measure point $q_{i j k h}$ such that both angles $\measuredangle_{q_{i j k h}}\left(p_{i}, p_{k}\right)$ and $\measuredangle_{q_{i j k h}}\left(p_{j}, p_{h}\right)$ are zero (modulo $\pi$ ). In fact this point is given by the intersection of the two diagonals $\overrightarrow{p_{i} p_{k}}$ and $\overrightarrow{p_{j} p_{h}}$. Then one performs the following construction: take the three tuples $\left(p_{1}, p_{2}, p_{3}, p_{4}\right)$, $\left(p_{1}, p_{2}, p_{5}, p_{4}\right)$ and $\left(p_{1}, p_{2}, p_{3}, p_{5}\right)$ and denote by $q_{1}, q_{2}$ and $q_{3}$ the points $q_{1234}, q_{1254}$ and $q_{1235}$ respectively, as in Fig. 3.

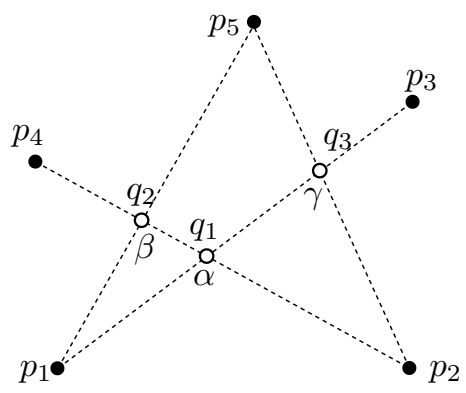

Figure 3. Identification of 5 points from the knowledge of their profile.

Since by hypothesis we know the profile of $p_{1}, \ldots, p_{5}$, then we know the angles $\alpha=\measuredangle_{q_{1}}\left(p_{1}, p_{2}\right)$, $\beta=\measuredangle_{q_{2}}\left(p_{1}, p_{2}\right)$ and $\gamma=\measuredangle_{q_{3}}\left(p_{1}, p_{2}\right)$ (modulo $\pi$ ). Then one sees that the angle $\measuredangle_{p_{5}}\left(p_{1}, p_{2}\right)$ is equal to $\beta+\gamma-\alpha$, namely it is completely determined by the profile. By symmetry, all angles $\measuredangle_{p_{k}}\left(p_{i}, p_{j}\right)$ are completely determined by the profile, hence the solution is unique up to similarities.

If 4 points are collinear, and the $5^{\text {th }}$ is not, then the above construction can still be applied to obtain enough angles, allowing to reconstruct the points up to similarity. 
Remark 4.2. For collinear target points, the situation is also quite clear: two collinear tuples of measure points have the same profile if and only if they are projectively equivalent.

Lemma 4.3. If $m \geq 4$, then the point identification problem from the co-profile has a unique solution.

Proof. Let $m \geq 4$ and let $q_{1}, \ldots, q_{m}$ be the measure points. Let $p_{1}$ be a target point. Assume that we know the co-profile surface $S^{\prime} \subset \mathbb{P}_{\mathbb{C}}^{m}$ of $\vec{q}=\left(q_{1}, \ldots, q_{m}\right)$ and $p_{1}$. Choose four points on $S^{\prime}$ with affine coordinates on the complex unit circle, general with respect to this property. Then the affine coordinates are the measurements of four additional target points $p_{2}, p_{3}, p_{4}, p_{5}$; by generality assumptions, we may assume that there is no cyclic cubic curve passing through all target and measure points and that the target points are neither collinear nor cocircular. The transpose of the matrix consisting of the four coordinate vectors is now considered as an input for profile interpolation. Since $m=4$, profile interpolation is uniquely solvable; let $S \subset \mathbb{P}_{\mathbb{C}}^{3}$ be the result. By Proposition 4.1, the profile determines $p_{1}, \ldots, p_{5}$ uniquely (up to similarity). The points $q_{1}, \ldots, q_{m}$ can then be obtained by the resection map (see the proof of Proposition 2.4).

Proposition 4.4. If $t=4$, then the point identification problem for the profile has, in general, two solutions.

Proof. Assume that $p_{1}, \ldots, p_{4}$ are unknown target point in general position with a known profile surface $S \subset \mathbb{P}_{\mathbb{C}}^{3}$. As in the previous proof, we choose four points on $S$ with affine coordinates on the complex unit circle, general with respect to this property. Their affine coordinates are the measurements from four unknown measurement points $q_{1}, \ldots, q_{4}$. We transpose the matrix consisting of these four coordinate vectors and get an input for the co-profile interpolation problem. By Proposition 3.5, we get two solutions $S^{\prime}$ and $S^{\prime \prime}$ in $\mathbb{P}_{\mathbb{C}}^{3}$. For each of these two, using Lemma 4.3 we can then identify the points $\vec{q}=\left(q_{1}, \ldots, q_{m}\right)$ and $p_{1}$ uniquely, up to similarity; by permuting target points, we can get also the remaining target points for both cases.

Notation. For more fluent language, we call a sequence of pairwise distinct 4 points a quadrilateral, and the second solution to its point identification problem its twin (which is determined up to similarity).

Remark 4.5. Here is a geometric construction for the twin quadrilateral. If the vertices of a quadrilateral $V$ are cocircular, then $V$ is its own twin (in other words, it can be recognized uniquely by point identification). Otherwise, the twin is obtained by constructing the centers of the circles that are circumscribed to the four triangles formed by the vertices of $V$ and then applying a reflection to this quadrilateral (see Fig. 4).

The proof of this statement is computational: we compute the profile for a quadrilateral with symbolic coordinates, and compare with the profile for the centers of the four circles above. This was done using the computer algebra system Maple ${ }^{3}$.

We summarize the results we obtained so far about point identification in the following theorem.

Theorem 4.6. Assume that $(t-3)(m-2) \geq 2$. The problem of identifying unknown target points $p_{1}, \ldots, p_{t}$ and measure points $q_{1}, \ldots, q_{m}$ from a given double angle matrix $M_{\vec{p}, \vec{q}}$ is uniquely solvable unless we are in the following cases:

- $t=4$ (here there are, in general, two solutions);

- $m=3$ (here there are, in general, two solutions);

\footnotetext{
${ }^{3}$ See http://www.risc.jku.at/people/jschicho/pub/twinmaple.html.
} 

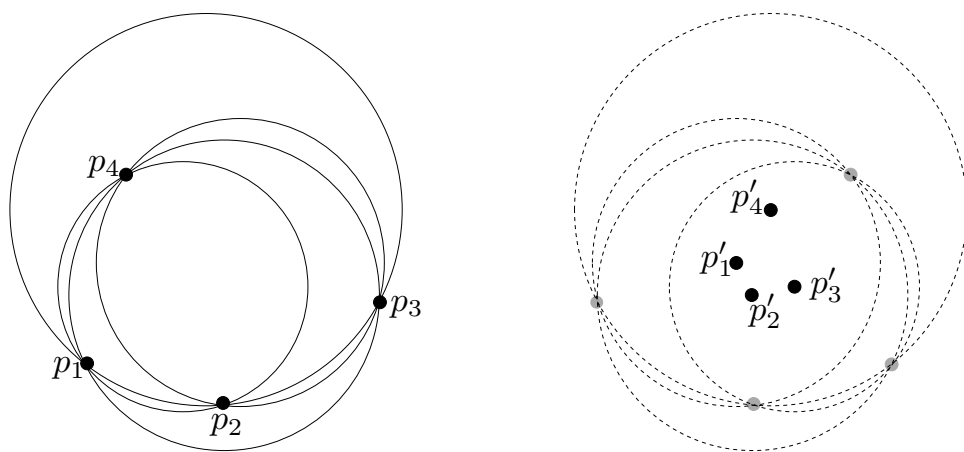

Figure 4. Construction of the twin quadrilateral: given $p_{1}, p_{2}, p_{3}$ and $p_{4}$ we consider the four circles passing through 3 out of the 4 points. The centers of these circles give the vertices $p_{1}^{\prime}, p_{2}^{\prime}, p_{3}^{\prime}$ and $p_{4}^{\prime}$ of the mirrored twin quadrilateral.

- all target and measure points lie on a cyclic cubic curve;

- at least 5 target points are cocircular or collinear;

- at least 4 measure points are cocircular or collinear.

\section{$5 \quad$ Ambiguities for directed angles}

In this last section we give a closer study of the case of 4 target points, and in particular we try to highlight some properties relating a quadrilateral to its twin.

The complex entries of the double angle matrix $M_{\vec{p}, \vec{q}}$ give conditions on the lines through $p_{i}$ and $q_{j}$ for $i \in\{1, \ldots, t\}$ and $j \in\{1, \ldots, m\}$, but they do not include information about the directions, i.e. on which of the two rays the point $p_{i}$ is lying. In a situation where we have two solutions for the point identification problem, it may still be possible to tell the right solution by taking direction information into account. However our final result (see Theorem 5.4) will make explicit that if measurements are taken from points in some prescribed region of the plane, then a quadrilateral cannot be distinguished from its twin even if we take directions into account.

Remark 5.1. If the point identification has infinitely many solutions, then there are always ambiguities even if we take direction information into account, because the direction information only allows to tell finitely many cases apart.

Let $\vec{p}=\left(p_{1}, \ldots, p_{4}\right)$ be a nondegenerated quadrilateral, i.e. a quadruple of points such that no three are collinear. Then from what we saw at the end of Section 4 there is a twin quadrilateral $\vec{p}^{\prime}=\left(p_{1}^{\prime}, \ldots, p_{4}^{\prime}\right)$ and both $\vec{p}$ and $\vec{p}^{\prime}$ have the same profile surface $S \subset \mathbb{P}_{\mathbb{C}}^{3}$. We define the twin map:

$$
\begin{aligned}
\rho_{\vec{p}, \vec{p}^{\prime}}: & \mathbb{R}^{2} \backslash\left\{p_{1}, \ldots, p_{4}\right\} \longrightarrow \mathbb{R}^{2}, \\
& q \mapsto \rho_{\vec{p}, \vec{p}^{\prime}}(q):=\left(f_{\vec{p}^{\prime}}^{-1} \circ f_{\vec{p}}\right)(q) .
\end{aligned}
$$

If $p_{1}, \ldots, p_{4}$ are cocircular, then the twin quadrilateral is similar to the original one and the twin map is just a composition of a rotation, a dilation and a translation. From now on, we assume that the points are not cocircular.

Algebraically, the twin map can be extended to a birational automorphism of $\mathbb{P}_{\mathbb{C}}^{1} \times \mathbb{P}_{\mathbb{C}}^{1}$. The exceptional curves partition the real plane minus the exceptional locus into open connected regions. Each open region is mapped homeomorphically to its image region. These image regions are the result of an analogous partition by the exceptional curves of $\rho_{\vec{p}^{\prime}, \vec{p}}$. This strongly motivates our interest in the exceptional curves of the twin map. They are described in the next proposition. 
Proposition 5.2. The exceptional curves of $\rho_{\vec{p}, \vec{p}^{\prime}}$ are the four circles passing through three of the points $p_{1}, \ldots, p_{4}$.

Proof. The base locus of the twin map is equal to the set $\left\{p_{1}, \ldots, p_{4}\right\}$. Because the image is a smooth surface, the exceptional curves are in $(-1)$-classes of the blowing up $\mathbb{Y}$ of $\mathbb{P}_{\mathbb{C}}^{1} \times \mathbb{P}_{\mathbb{C}}^{1}$ at $p_{1}, \ldots, p_{4}$. The class group of $\mathbb{Y}$ is freely generated by the class $L_{1}$ of bidegree $(1,0)$, the class $L_{2}$ of bidegree $(0,1)$, and the four exceptional classes $E_{1}, E_{2}, E_{3}, E_{4}$ of the blowing up map. Here is the list of all $(-1)$-classes in the class group of $\mathbb{Y}$ :

- $E_{1}, \ldots, E_{4}$, corresponding to the four base points;

- $L_{1}-E_{1}, \ldots, L_{1}-E_{4}, L_{2}-E_{1}, \ldots, L_{2}-E_{4}$, corresponding to the fibers of the two projections passing through the base points;

- $L_{1}+L_{2}-E_{2}-E_{3}-E_{4}, \ldots, L_{1}+L_{2}-E_{1}-E_{2}-E_{3}$, corresponding to the circles passing through three of the four base points.

This list is very well known, since the blow up of $\mathbb{P}_{\mathbb{C}}^{1} \times \mathbb{P}_{\mathbb{C}}^{1}$ at four points is isomorphic to the blow up of $\mathbb{P}_{\mathbb{C}}^{2}$ at five points. Hence $\mathbb{Y}$ is a Del Pezzo surface of degree 4 , and it contains exactly the 16 (-1)-classes listed before (for a reference, see [7, Theorems 24.3, 24.4 and 24.5] or [4, Chapter 8 and in particular the remark following Lemma 8.2.22]). In the factorization of $\rho_{\vec{p}, \vec{p}^{\prime}}$ into blowing ups and blowing downs, we have four blowing ups, so we also need to have four blowing downs. The only choice of four (-1)-classes not containing the exceptional classes of the blowing up, and such that it is symmetric under permutations of $E_{1}, \ldots, E_{4}$ and under permutations of $L_{1}$ and $L_{2}$, is the choice of the four circles.

We denote by $C_{1}, \ldots, C_{4}$ the four circles mentioned in Proposition 5.2, where $C_{i}$ is the circle that does not pass through $p_{i}$, for $i \in\{1, \ldots, 4\}$. We call them the fundamental circles of $\vec{p}$.

The following proposition is not really needed; we just mention it because it is a nice description of the structure of the partition by fundamental circles.

Proposition 5.3. The complement of the fundamental circles of a quadrilateral $\vec{p}$ has 10 connected components. Four of them are delimited by three circles, and the other 6 are delimited by two circles. The boundary of the unique unbounded region is constituted by 2 arcs if and only if the quadrilateral is convex (see Fig. 5).

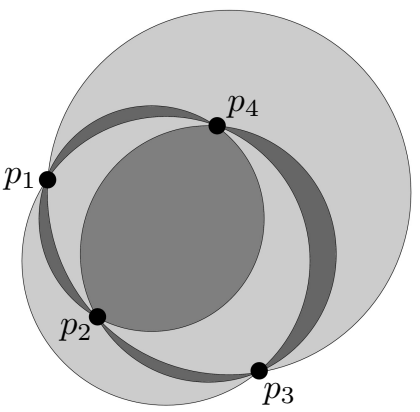

(a)

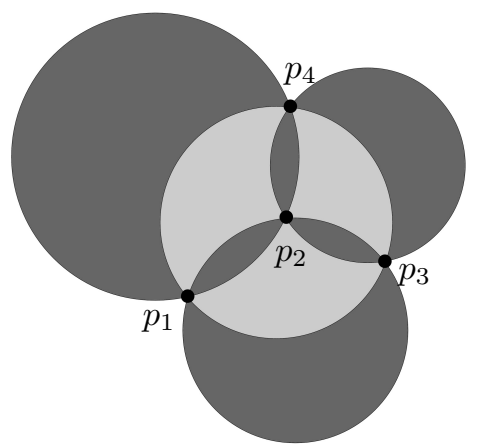

(b)

Figure 5. The 10 regions forming the complement of the fundamental circles of a quadrilateral: in the convex case (a) the boundary of the unbounded region is given by 2 arcs, while in the concave case (b) the boundary is given by 3 arcs.

If $\vec{p}$ is convex, then the unique region containing the intersection of the inner diagonals is called the inner region. It is important to observe that the twin of a convex quadrilateral is again convex, and the twin map maps the inner region to the inner region. 
Theorem 5.4. Let $\vec{p}$ be a nondegenerate convex quadrilateral and assume that all measure points $q_{1}, \ldots, q_{m}$ (where $m$ is arbitrary) are contained in the inner region. Let $\vec{p}^{\prime}$ be the twin quadrilateral of $\vec{p}$ and set $q_{i}^{\prime}=\rho_{\vec{p}, \vec{p}^{\prime}}\left(q_{i}\right)$ for $i \in\{1, \ldots, m\}$. Then we have

$$
\measuredangle_{q_{j}}\left(p_{1}, p_{i}\right)=\measuredangle_{q_{j}^{\prime}}\left(p_{1}^{\prime}, p_{i}^{\prime}\right)
$$

for all $i \in\{2, \ldots, t\}$ and $j \in\{1, \ldots, m\}$.

Proof. The construction guarantees that $M_{\vec{p}, \vec{q}}=M_{\vec{p}^{\prime}, \vec{q}^{\prime}}$. Hence for all $i \in\{2, \ldots, t\}$ and $j \in\{1, \ldots, m\}$ we have the equality $\left(\measuredangle_{q_{j}}\left(p_{1}, p_{i}\right)\right)^{2}=\left(\measuredangle_{q_{j}^{\prime}}\left(p_{1}^{\prime}, p_{i}^{\prime}\right)\right)^{2}$, where we represent angles by complex numbers, so that doubling the angle corresponds to squaring the complex representation. Hence the quotient of left hand side and right hand side is \pm 1 . We consider this quotient as a function $\phi$ from the complement of the fundamental circles to $\{1,-1\}$. It is easy to see that this map is continuous, therefore constant on any region, since they are connected. At the intersection of the inner diagonals the map $\phi$ assumes the value 1 , therefore it is constantly equal to 1 on the whole inner region. From this the thesis follows.

\section{Acknowledgements}

We thank Kristian Ranestad for pointing out to us the solution of a crucial interpolation problem, Bert Jüttler and Martin Peternell for helping us to orient ourselves in the literature on geodesy, and the anonymous referees for helpful comments and pointers to literature on photogrammetry. The authors' research is supported by the Austrian Science Fund (FWF): W1214-N15/DK9 and P26607 - "Algebraic Methods in Kinematics: Motion Factorisation and Bond Theory".

\section{References}

[1] Åström K., Oskarsson M., Solutions and ambiguities of the structure and motion problem for 1D retinal vision, J. Math. Imaging Vision 12 (2000), 121-135.

[2] Carlsson S., Weinshall D., Dual computation of projective shape and camera position from multiple images, Int. J. Computer Vision 27 (1998), 227-241.

[3] Cox D.A., Equations of parametric curves and surfaces via syzygies, in Symbolic Computation: Solving Equations in Algebra, Geometry, and Engineering (South Hadley, MA, 2000), Contemp. Math., Vol. 286, Amer. Math. Soc., Providence, RI, 2001, 1-20, math.AG/0010107.

[4] Dolgachev I.V., Classical algebraic geometry. A modern view, Cambridge University Press, Cambridge, 2012.

[5] Eisenbud D., Harris J., On varieties of minimal degree (a centennial account), in Algebraic geometry, Bowdoin, 1985 (Brunswick, Maine, 1985), Proc. Sympos. Pure Math., Vol. 46, Amer. Math. Soc., Providence, RI, 1987, 3-13.

[6] Eisenbud D., Popescu S., The projective geometry of the Gale transform, J. Algebra 230 (2000), 127-173, math.AG/9807127.

[7] Manin Yu.I., Cubic forms. Algebra, geometry, arithmetic, North-Holland Mathematical Library, Vol. 4, 2nd ed., North-Holland Publishing Co., Amsterdam, 1986.

[8] Mooers R.L., Finding your way in the outdoors: compass navigation, map reading, route finding, weather forecasting, E.P. Dutton, 1972.

[9] Oskarsson M., Solution and ambiguities for structure and motion problems, Ph.D. Thesis, Lund University, 2002.

[10] Quan L., Two-way ambiguity in 2D projective reconstruction from three uncalibrated 1D images, IEEE Trans. Pattern Analysis Mach. Int. 23 (2001), 212-216. 